\title{
Uniqueness of weakly weighted-sharing a small function by a meromorphic function and its differential polynomial
}

Harina P. Waghamore and Ramya Maligi

Department of Mathematics, Jnanabharathi Campus, Bangalore University, Bengaluru-560056, India

Received: 3 November 2017, Accepted: 17 January 2018

Published online: 9 April 2018.

Abstract: In this paper we study the uniqueness of weakly weighted-sharing a small function by a meromorphic function and its differential polynomial. The result of the paper improve some recent results due to Hong-Yan Xu and Yi Hu [5].

Keywords: Meromorphic function, shared value, small function, weakly-sharing.

\section{Introduction}

Let $f$ be a meromorphic function in the open complex plane $\mathbb{C}$. We use the standard notations of Nevanlinna theory, which can be found in [7]. We denote by $S(r, f)$ any quantity satisfying $S(r, f)=o\{T(r, f)\}$ as $r \rightarrow \infty$ possibly outside a set of finite linear measure.

A meromorphic function $a=a(z)$ is called a small function of $f$ if $T(r, a)=S(r, f)$. We denote by $S(f)$ the collection of all small functions of $f$. Clearly $\mathbb{C} \subset S(f)$.

Let $f$ and $g$ be two meromorphic functions in $\mathbb{C}$ and $a \in S(f) \cap S(g)$. We say that $f$ and $g$ share the function $a=a(z)$ CM (counting multiplicities) or IM (ignoring multiplicities) if $f-a$ and $g-a$ have the same set of zeros counting multiplicities or ignoring multiplicities respectively.

Definition 1. [5] Let $k$ be a positive integer, and let $f$ be a meromorphic function and $a \in S(f)$.

(i) $\bar{N}(r, a ; f \mid \geq k)$ denotes the counting function of zeros of $f-a$ whose multiplicities are not less than $k$, where each zero is counted only once.

(ii) $\bar{N}(r, a ; f \mid \leq k)$ denotes the counting function of zeros of $f-a$ whose multiplicities are not greater than $k$, where each zero is counted only once.

(iii) $N_{p}(r, a ; f)=\bar{N}(r, a ; f)+\sum_{k=2}^{p} \bar{N}(r, a ; f \mid \geq k)$.

Definition 2. [2] For any complex number $c \in \mathbb{C} \cup\{\infty\}$, we denote by $\delta_{p}(c, f)$ the quantity

$$
\delta_{p}(c, f)=1-\lim _{r \rightarrow \infty} \sup \frac{N_{p}(r, c ; f)}{T(r, f)},
$$


where $p$ is a positive integer. Clearly $\delta_{p}(c, f) \geq \delta(c, f)$.

Let $N_{E}(r, a)$ be the counting function of all common zeros of $f-a$ and $g-a$ with the same multiplicities, and $N_{0}(r, a) b e$ the counting functions of all common zeros of $f-a$ and $g-a$ ignoring multiplicities. Denotes by $\bar{N}_{E}(r, a)$ and $\bar{N}_{0}(r, a)$ the reduced counting functions of $f$ and $g$ corresponding to the counting functions $N_{E}(r, a)$ and $N_{0}(r, a)$, respectively. If

$$
\bar{N}(r, a ; f)+\bar{N}(r, a ; g)-2 \bar{N}_{E}(r, a)=S(r, f)+S(r, g),
$$

then we say that $f$ and $g$ share a "CM". If

$$
\bar{N}(r, a ; f)+\bar{N}(r, a ; g)-2 \bar{N}_{0}(r, a)=S(r, f)+S(r, g)
$$

then we say that $f$ and $g$ share a "IM".

Definition 3. [5] Let $f$ and $g$ be two nonconstant meromorphic functions sharing a "IM", for a $\in S(f) \cap S(g)$, and a positive integer $k$ or $\infty$.

(i) $\bar{N}_{E}^{k)}(r, a)$ denotes the counting function of zeros of $f-a$ whose multiplicities are equal to the corresponding zeros of $g-a$, both of their mutiplicities are not greater than $k$, where each zero is counted only once.

(ii) $\bar{N}_{0}^{(k}(r, a)$ denotes the reduced counting function of zeros of $f-a$ which are zeros of $g-a$, both of their mutiplicities are not less than $k$, where each zero is counted only once.

(iii) Let $z_{0}$ be the zeros of $f-a$ with multiplicity $p$ and zeros of $g-a$ with multiplicity $q$. Denote by $\bar{N}_{f>k}(r, a$; $g)$ the reduced counting function of those zeros of $f-a$ and $g-a$ such that $p>q=k \cdot \bar{N}_{g>k}(r, a ; g)$ is defined analogously.

(iv) $\bar{N}_{*}(r, a ; f, g)$ denotes the reduce counting function of zeros of $f$-a whose multiplicities differ from the multiplicities of the corresponding zeros of $g-a$.

Clearly,

$\bar{N}_{*}(r, a ; f, g)=\bar{N}_{*}(r, a ; g, f)$ and $\bar{N}_{*}(r, a ; f, g)=\bar{N}_{L}(r, a ; f)+\bar{N}_{L}(r, a ; g)$.

Definition 4. [5] For $a \in S(f) \cap S(g)$, if $k$ is a positive integer or $\infty$, and

$$
\begin{aligned}
& \bar{N}(r, a ; f \mid \leq k)-\bar{N}_{E}^{k)}(r, a)=S(r, f), \bar{N}(r, a ; f \mid \geq k+1)-\bar{N}_{0}^{(k+1}(r, a)=S(r, f) \\
& \bar{N}(r, a ; g \mid \leq k)-\bar{N}_{E}^{k)}(r, a)=S(r, g), \bar{N}(r, a ; g \mid \geq k+1)-\bar{N}_{0}^{(k+1}(r, a)=S(r, g),
\end{aligned}
$$

or if $k=0$ and

$$
\bar{N}(r, a ; f)-\bar{N}_{0}(r, a)=S(r, f), \bar{N}(r, a ; g)-\bar{N}_{0}(r, a)=S(r, g),
$$

where $\bar{N}_{0}(r, a)$ is the reduce counting functions of all common zeros $f-a$ and $g-a$ ignoring multiplicities, then we say $f$ and $g$ weakly share a with weight $k$. Here, we write $f, g$ share " $(a, k)$ " to mean that $f, g$ weakly share a with weight $k$.

Obviously, if $f$ and $g$ share " $(a, k)$ ", then $f$ and $g$ share " $(a, p)$ " for any $p(0 \leq p \leq k)$. Also, we note that $f$ and $g$ share a "IM" or "CM" if and only if $f$ and $g$ share " $(a, 0)$ " or " $(a, \infty)$ ", respectively.

Definition 5. [5] Let

$$
L(f)=f^{(n)}+a_{n-1} f^{(n-1)}+\ldots+a_{0} f
$$

be a differential polynomial of $f$, where $a_{j}(j=0,1, \ldots, n-1) \in S(f)$.

In 2003, Yu [8] considered the uniqueness problem of an entire function or meromorphic function when it shares one small function with its derivative and proved the following results. 
Theorem 1. Let $n \geq 1$, let $f$ be a non-constant entire function, $a \in S(f)$ and $a \neq 0, \infty$. If $f, f^{(n)}$ share $a C M$ and $\delta(0, f)>\frac{3}{4}$, then $f \equiv f^{(n)}$.

Theorem 2. Let $n \geq 1$, let $f$ be a non-constant non-entire meromorphic function, $a \in S(f)$ and $a \not \equiv 0, \infty, f$ and $a$ do not have any common pole. If $f, f^{(n)}$ share $a C M$ and $4 \delta(0, f)+2(8+n) \Theta(\infty, f)>19+2 n$, then $f \equiv f^{(n)}$.

In 2004, Liu and Gu [3] applied a different method and obtained the following results.

Theorem 3. Let $f$ be a non-constant meromorphic function, $a \in S(f)$ and $a \neq 0, \infty$. If $f, f^{(n)}$ share a CM, $f$ and a do not have any common pole of same multiplicity and $2 \delta(0, f)+4 \Theta(\infty, f)>5$, then $f \equiv f^{(n)}$.

Theorem 4. Let $n \geq 1$, let $f$ be a non-constant entire function, $a \in S(f)$ and $a \neq 0, \infty$. If $f, f^{(n)}$ share $a C M$ and $\delta(0, f)>\frac{1}{2}$, then $f \equiv f^{(n)}$.

In 2011, Hong-Yan Xu and Yi Hu [5] obtained the following result which improve the results of [15, 8].

Theorem 5. Let $n \geq 1$, let $f$ be a non-constant meromorphic function, $a \in S(f)$ and $a \neq \equiv, \infty$. Suppose that $L(f)$ is defined by $(*)$, If $f, L(f)$ share " $(a, k)$ ". Then $f \equiv L(f)$ if one of the following assumptions holds,

1. $2 \leq k \leq \infty$ and

$$
4 \Theta(\infty, f)+2 \delta_{2+n}(0, f)>5
$$

2. $k=1$ and

$$
\left(\frac{7}{2}+n\right) \Theta(\infty, f)+\frac{3}{2} \delta_{2}(0, f)+\delta_{2+n}(0, f)>n+5
$$

3. $k=0$ and

$$
(6+2 n) \Theta(\infty, f)+\delta_{2}(0, f)+2 \Theta(0, f)+2 \delta_{2+n}(0, f)>(2 n+10) .
$$

We define a monomial $M[f]$ and differential polynomial $H[f]$ as follows,

Let $p_{0}, p_{1}, \ldots, p_{k}$ be non-negative integers. We call

$$
M[f]=f^{p_{0}}\left(f^{\prime}\right)^{p_{1}} \ldots\left(f^{(k)}\right)^{p_{k}}
$$

a differential monomial in $f$ with degree $d_{M}=p_{0}+p_{1}+\ldots+p_{k}$ and weight $\Gamma_{M}=p_{0}+2 p_{1}+\ldots+(k+1) p_{k}$, and

$$
H[f]=\sum_{j=1}^{n} a_{j} M_{j}[f]
$$

where $a_{j}$ are small functions of $f$, is called a differential polynomial in $f$ of degree $d=\max \left\{d_{M_{j}}, 1 \leq j \leq n\right\}$ and weight $\Gamma=\max \left\{\Gamma_{M_{j}}, 1 \leq j \leq n\right\}$, furthermore if $\operatorname{deg}\left(M_{j}\right)=d(j=1,2, \ldots, n)$, then $H[f]$ is a homogeneous differential polynomial in $f$ of degree $d$.

In this paper, we improve the above Theorems and obtain the following results.

Theorem 6. Let $f$ be a non-constant meromorphic function and $H[f]$ be a non-constant homogeneous differential polynomial of degree $d$ and weight $\Gamma$ satisfying $\Gamma \geq(k+2) d-2$. Let $a(z) \in S(f)$ be a small meromorphic function of $f$ such that $a(z) \not \equiv 0, \infty$. Suppose that $f-a$ and $H[f]-$ a share $(0, k)$. Then $\frac{H[f]-a}{f-a}=C$ for some non-zero constant $C$ if one of the following asumptions holds,

(i) $2 \leq k \leq \infty$ and

$$
4 \Theta(\infty, f)+\delta_{2}(0, f)+d \delta_{2+\Gamma-d}\left(0, f^{d}\right)>5
$$

(ii) $k=1$ and

$$
\left(\frac{7}{2}+\Gamma-d\right) \Theta(\infty, f)+\frac{3}{2} \delta_{2}(0, f)+d \delta_{2+\Gamma-d}\left(0, f^{d}\right)>\Gamma+4
$$

(iii) $k=0$ and

$$
(6+2 \Gamma-2 d) \Theta(\infty, f)+\delta_{2}(0, f)+2 \Theta(0, f)+d \delta_{1+\Gamma-d}\left(0, f^{d}\right)+d \delta_{2+\Gamma-d}\left(0, f^{d}\right)>2 \Gamma+9 .
$$


Especially, when $k=0$, i.e., $f$ and $H$ share $a$ IM, if (4) holds, then $f \equiv H[f]$.

From Theorem 6 we have the following corollary.

Corollary 1. Let $f$ be a non-constant entire function and $a \equiv a(z)(\not \equiv 0, \infty)$ be a meromorphic function such that $T(r, a)=$ $S(r, f)$. If $f, H[f]$ share " $(a, k)$ ", $k \geq 2$ and $\delta_{2+\Gamma-d}\left(0, f^{d}\right)>\frac{1}{d+1}$, or if $f, H[f]$ share " $(a, 1)$ " and $\delta_{2+\Gamma-d}\left(0, f^{d}\right)>\frac{2 d+1}{3+2 d}$, or if $f, H[f]$ share " $(a, 0)$ " and $\delta_{2+\Gamma-d}\left(0, f^{d}\right)>\frac{2 d+2}{d}-\frac{1}{d}\left(\delta_{2}(0, f)+2 \Theta(0, f)+d \delta_{1+\Gamma-d}\left(0, f^{d}\right)\right)$, then $\frac{H[f]-a}{f-a}=C$ for some non-zero constant $C$ and $f \equiv H[f]$ for $k=0$, where $H[f]$ is defined by (1).

\section{Some lemmas}

For the proof of our main results, we need the following lemmas.

Lemma 1. [4] Let $H[f]$ be a non-constant differential polynomial. Let $z_{0}$ be a pole of $f$ order $p$ and neither a zero nor a pole of coefficients of $H[f]$. Then $z_{0}$ is a pole of $H[f]$ with order at most $p d+(\Gamma-d)$.

Lemma 2. [4] Let $f$ be a non-constant meromorphic function, $H[f]$ is a homogeneous differential polynomial in $f$ of degree $d$ and weight $\Gamma$, and let $p$ be a positive integer. If $H[f] \not \equiv 0$ and $\Gamma \geq(k+2) d-(p+1)$, we have

$$
\begin{gathered}
N_{p}\left(r, \frac{1}{H}\right) \leq T(r, H)-d T(r, f)+N_{p+\Gamma-d}\left(r, \frac{1}{f^{d}}\right)+S(r, f), \\
N_{p}\left(r, \frac{1}{H}\right) \leq(\Gamma-d) \bar{N}(r, f)+N_{p+\Gamma-d}\left(r, \frac{1}{f^{d}}\right)+S(r, f) .
\end{gathered}
$$

Lemma 3. [6] Let $k$ be a nonnegative integer or $\infty, F$ and $G$ be two nonconstant meromorphic functions, $F$ and $G$ share "(1,k)". Let

$$
\Delta=\left(\frac{F^{\prime \prime}}{F^{\prime}}-2 \frac{F^{\prime}}{F-1}\right)-\left(\frac{G^{\prime \prime}}{G^{\prime}}-2 \frac{G^{\prime}}{G-1}\right) .
$$

If $\Delta \not \equiv 0,2 \leq k \leq \infty$, then

$$
T(r, F) \leq N_{2}(r, \infty ; F)+N_{2}(r, 0 ; F)+N_{2}(r, \infty ; G)+N_{2}(r, 0 ; G)+S(r, F)+S(r, G) .
$$

The same inequalities holds for $T(r, G)$.

When $f$ and $g$ share 1 "IM", $\bar{N}_{L}(r, 1 ; f)$ denotes the counting function of the 1-points of $f$ whose multiplicities are greater than 1-points of $g$, where each zero is counted only once. Similarly, we denote $\bar{N}_{L}(r, 1 ; g), N_{E}^{1)}(r, 1 ; f)$ denotes the counting function of those simple 1-points of $f$ and $g$, and $\bar{N}_{E}^{(2}(r, 1 ; f)$ denotes the counting function of those multiplicity 1-points of $f$ and $g$, each point in these counting functions is counted only once. In the same way, one can define $N_{E}^{1)}(r, 1 ; g), \bar{N}_{E}^{(2}(r, 1 ; g)$.

Lemma 4. [5] If $f$, g be two nonconstant meromorphic functions such that they share "(1,1)", then

$$
2 \bar{N}_{L}(r, 1 ; f)+2 \bar{N}_{L}(r, 1 ; g)+\bar{N}_{E}^{(2}(r, 1 ; f)-\bar{N}_{f>2}(r, 1 ; g) \leq N(r, 1 ; g)-\bar{N}(r, 1 ; g) .
$$

Lemma 5. [5] Let $f$, g share “(1,1)". Then

$$
\bar{N}_{f>2}(r, 1 ; g) \leq \frac{1}{2} \bar{N}(r, 0 ; f)+\frac{1}{2} \bar{N}(r, \infty ; f)-\frac{1}{2} \bar{N}_{0}\left(r, 0, f^{\prime}\right)+S(r, f) .
$$


Lemma 6. [5] Let $f$ and $g$ be two nonconstant meromorphic functions sharing "(1,0)". Then

$$
\bar{N}_{L}(r, 1 ; f)+2 \bar{N}_{L}(r, 1 ; g)+\bar{N}_{E}^{(2}(r, 1 ; f)-\bar{N}_{f>1}(r, 1 ; g)-\bar{N}_{g>1}(r, 1 ; f) \leq N(r, 1 ; g)-\bar{N}(r, 1 ; g) .
$$

Lemma 7. [5] Let $f$, g share "(1,0)". Then

$$
\bar{N}_{L}(r, 1 ; f) \leq \bar{N}(r, 0 ; f)+\bar{N}(r, \infty ; f)+S(r, f) .
$$

Lemma 8. [5] Let $f$, g share "(1,0)". Then

(i) $\bar{N}_{f>1}(r, 1 ; g) \leq \bar{N}(r, 0 ; f)+\bar{N}(r, \infty ; f)-\bar{N}_{0}\left(r, 0, f^{\prime}\right)+S(r, f)$;

(ii) $\bar{N}_{g>1}(r, 1 ; f) \leq \bar{N}(r, 0 ; g)+\bar{N}(r, \infty ; f)-\bar{N}_{0}\left(r, 0, f^{\prime}\right)+S(r, g)$.

Proof. (proof of Theorem 6.) Let

$$
F=\frac{f}{a}, \quad G=\frac{H[f]}{a} .
$$

From the conditions of Theorem 6, we know that $F$ and $G$ share " $(1, k)$ ", and from (8), we have

$$
\begin{gathered}
T(r, F)=T(r, f)+S(r, f), T(r, G)=O(T(r, f))+S(r, f) . \\
\bar{N}(r, \infty ; F)=\bar{N}(r, \infty ; G)+S(r, f) .
\end{gathered}
$$

It is obvious that $f$ is a transcendental meromorphic function. Let $\Delta$ be defined by (7). We distinguish two cases

Case 1. $\Delta \equiv 0$. integrating (7), yields

$$
\frac{1}{F-1}=\frac{C}{G-1}+D
$$

where $C$ and $D$ are constants and $C \neq 0$. If there exists a pole $z_{0}$ of $f$ with multiplicity $p$ which is not zero or pole of $a$, then $z_{0}$ is a pole of $G$ with multiplicity $p d+(\Gamma-d)$, a pole of $F$ with multiplicity $p$. This contradicts (11) as $H$ contains at least one derivative. Therefore, we have

$$
\bar{N}(r, \infty ; F)=\bar{N}(r, \infty ; G)=\bar{N}(r, \infty ; f)=S(r, f) .
$$

(11) also shows that $F$ and $G$ share the value $1 \mathrm{CM}$. Next, we will prove $D=0$. Suppose $D \neq 0$, then we have

$$
\frac{1}{F-1}=\frac{D\left(G-1+\frac{C}{D}\right)}{G-1}
$$

So, we have

$$
\bar{N}\left(r, 0 ; D\left(G-1+\frac{C}{D}\right)\right)=\bar{N}\left(r, \infty ; \frac{F-1}{G-1}\right)=S(r, f) .
$$

Subcase 1.1. If $\frac{C}{D} \neq 1$, then by using (12), (14) and the second fundamental theorem, we have

$$
\begin{aligned}
T(r, F) & \leq \bar{N}(r, \infty ; G)+\bar{N}(r, 0 ; G)+\bar{N}\left(r, 0 ; G-1+\frac{C}{D}\right)+S(r, F) \\
& \leq \bar{N}(r, 0 ; G)+S(r, F) \leq(1+o(1)) T(r, G) .
\end{aligned}
$$

This gives that

$$
T(r, G)=\bar{N}(r, 0 ; G)+S(r, F)=N_{1}(r, 0 ; G)+S(r, F) .
$$

So we have

$$
T(r, H)=N(r, 0 ; H)+S(r, f)=N_{1}(r, 0 ; H)+S(r, f) .
$$


Let $p=1$, then from assumption we have

$$
\Gamma \geq(k+2) d-2=(k+2) d-(p+1) .
$$

Thus from (5) in Lemma 2, we get

$$
T(r, H)=N_{1}(r, 0 ; H)+S(r, f) \leq T(r, H)-d T(r, f)+N_{1+\Gamma-d}\left(r, 0 ; f^{d}\right)+S(r, f) .
$$

So we have

$$
d T(r, f) \leq N_{1+\Gamma-d}\left(r, 0 ; f^{d}\right)+S(r, f) .
$$

This gives that

$$
d T(r, f)=N_{1+\Gamma-d}\left(r, 0 ; f^{d}\right)+S(r, f)
$$

So we have

$$
\delta_{2+\Gamma-d}\left(r, 0 ; f^{d}\right)=\delta_{1+\Gamma-d}\left(r, 0 ; f^{d}\right)=0 .
$$

Since (12), we get

$$
\Theta(\infty, f)=1 .
$$

Subcase 1.2. $k \geq 2$. By using (2) and the definition of deficiency, we get a contradiction.

Subcase 1.3. $k=1$. By using (3) and the definition of deficiency, we get a contradiction.

Subcase 1.4. $k=0$. By using (4) and the definition of deficiency, we get a contradiction.

Subcase 1.5. If $\frac{C}{D}=1$, then from (13), we have

$$
\frac{1}{F-1} \equiv C \frac{G}{G-1}
$$

This gives us that

$$
\left(F-1-\frac{1}{C}\right) G \equiv-\frac{1}{C}
$$

Using that $F=\frac{f}{a}$ and $G=\frac{H}{a}$, we get

$$
f-\left(a+\frac{1}{C}\right) \equiv-\frac{a^{2}}{C} \cdot \frac{1}{H} .
$$

Using (12), (16), Lemma 1 and the first fundamental theorem, we get

$$
\begin{aligned}
(d+1) T(r, f) & =T\left(r, 0 ; f^{d}\left(f-\left(1+\frac{1}{C}\right) a\right)\right)+O(1) \\
& =T\left(r, \infty ;-\frac{C H}{f^{d} a^{2}}\right)+O(1) \\
& =N\left(r, \infty ; \frac{H}{f^{d}}\right)+S(r, f) \\
& \leq d N(r, 0 ; f)+S(r, f) \\
& \leq(d+o(1)) T(r, f),
\end{aligned}
$$

which is a contradiction, hence $D=0$. This gives from (11) that

$$
\frac{G-1}{F-1} \equiv C
$$


So we get $\frac{H[f]-a}{f-a}=C(C \neq 0$.) Next, we will prove $C=1$ when $l=0$. Suppose $C \neq 1$, then we have

$$
F \equiv \frac{1}{C}(G-1+C)
$$

and

$$
N(r, 0 ; F)=N(r,(1+C) ; G) .
$$

By the second fundamental theorem and (12) (17), we have

$$
\begin{aligned}
T(r, G) & \leq \bar{N}(r, \infty ; G)+\bar{N}(r, 0 ; G)+\bar{N}(r,(1+C) ; G)+S(r, f) \\
& \leq \bar{N}(r, 0 ; G)+\bar{N}(r, 0 ; F)+S(r, f) \\
& =N_{1}(r, 0 ; G)+\bar{N}(r, 0 ; F) .
\end{aligned}
$$

By Lemma 2 for $p=1$, we have

$$
d T(r, f) \leq N_{1+\Gamma-d}\left(r, 0 ; f^{d}\right)+\bar{N}(r, 0 ; f)+S(r, f) .
$$

From the above formula and the definition of deficiency, we have

$$
d \delta_{1+\Gamma-d}\left(0, f^{d}\right)+\Theta(0, f) \leq 1
$$

So we have

$$
d \delta_{2+\Gamma-d}\left(0, f^{d}\right)+\delta_{2}(0, f) \leq 1, d \delta_{1+\Gamma-d}\left(0, f^{d}\right) \leq 1 .
$$

Combining (18) (19) (15) with the assumptions of Theorem 6, we get a contradiction. So $C=1$ and $F \equiv G$, i.e. $f \equiv H[f]$. This is just the conclusion of this theorem.

Case 2. $\Delta \not \equiv 0$.

Subcase 2.1. $k \geq 2$. It follows from Lemma 3 that

$$
T(r, G) \leq N_{2}(r, \infty ; F)+N_{2}(r, 0 ; F)+N_{2}(r, \infty ; G)+N_{2}(r, 0 ; G)+S(r, F)+S(r, G) .
$$

Noting that

$$
N_{2}(r, 0 ; G)=N_{2}\left(r, 0 ; \frac{H}{a}\right) \leq N_{2}(r, 0 ; H)+S(r, f) .
$$

Let $p=2$, then from assumption we have

$$
\Gamma \geq(k+2) d-2>(k+2) d-(p+1) .
$$

Thus, from (5) in Lemma 2 we obtain that

$$
T(r, H) \leq 4 \bar{N}(r, \infty ; f)+N_{2}(r, 0 ; f)+T(r, H)-d T(r, f)+N_{2+\Gamma-d}\left(r, 0 ; f^{d}\right)+S(r, f) .
$$

So we have

$$
d T(r, f) \leq 4 \bar{N}(r, \infty ; f)+N_{2}(r, 0 ; f)+N_{2+\Gamma-d}\left(r, 0 ; f^{d}\right)+S(r, f) .
$$

This gives that

$$
4 \Theta(\infty, f)+\delta_{2}(0, f)+d \delta_{2+\Gamma-d}\left(0, f^{d}\right) \leq 5 .
$$

Which contradicts the assumption (2) of Theorem 6.

Subcase 2.2. $k=1$. We know that $F, G$ share " $(1,1)$ ", hence we have 


$$
\begin{aligned}
N(r, \infty ; H) & \leq \bar{N}(r, \infty ; F)+\bar{N}(r, 1 ; F \mid \geq 2)+\bar{N}(r, 0 ; F \mid \geq 2)+\bar{N}(r, 0 ; G \mid \geq 2) \\
& +\bar{N}_{0}\left(r, 0 ; F^{\prime}\right)+\bar{N}_{0}\left(r, 0 ; G^{\prime}\right)+S(r, f),
\end{aligned}
$$

and

$$
N(r, 1 ; F \mid=1) \leq N(r, 0 ; H)+S(r, f) \leq N(r, \infty ; H)+S(r, f),
$$

where $\bar{N}_{0}\left(r, 0 ; F^{\prime}\right)$ is the reduced counting function of those zeros of $F^{\prime}$ which are not the zeros of $F(F-1)$, and $\bar{N}_{0}\left(r, 0 ; G^{\prime}\right)$ is similarly defined. By the second fundamental theorem, we see that

$$
\begin{aligned}
T(r, F)+T(r, G) & \leq \bar{N}(r, \infty ; F)+\bar{N}(r, 0 ; F)+\bar{N}(r, \infty ; G)+\bar{N}(r, 0 ; G) \\
& +\bar{N}(r, 1 ; F)+\bar{N}(r, 1 ; G)-\bar{N}_{0}\left(r, 0 ; F^{\prime}\right) \\
& -\bar{N}_{0}\left(r, 0 ; G^{\prime}\right)+S(r, F)+S(r, G) .
\end{aligned}
$$

Using Lemmas (4)and (5), (21) and (22) we can get

$$
\begin{aligned}
\bar{N}(r, 1 ; F)+\bar{N}(r, 1 ; G) & \leq N(r, 1 ; F \mid=1)+\bar{N}_{L}(r, 1 ; F)+\bar{N}_{L}(r, 1 ; G)+\bar{N}_{E}^{(2}(r, 1 ; F)+\bar{N}(r, 1 ; G) \\
& \leq N(r, 1 ; F \mid=1)+N(r, 1 ; G)-\bar{N}_{L}(r, 1 ; F)-\bar{N}_{L}(r, 1 ; G)+\bar{N}_{F>2}(r, 1 ; G) \\
& \leq \bar{N}(r, 0 ; F \mid \geq 2)+\bar{N}(r, 0 ; G \mid \geq 2)+\bar{N}(r, \infty ; F)+\bar{N}_{*}(r, 1 ; F, G)+T(r, G) \\
& -m(r, 1 ; G)+O(1)+\frac{1}{2} \bar{N}(r, \infty ; F)-\bar{N}_{L}(r, 1 ; F)-\bar{N}_{L}(r, 1 ; G)+\frac{1}{2} \bar{N}(r, 0 ; F) \\
& +N_{0}\left(r, 0 ; F^{\prime}\right)+N_{0}\left(r, 0 ; G^{\prime}\right)+S(r, F)+S(r, G) .
\end{aligned}
$$

Combining (23) and (24), we can obtain

$$
\begin{aligned}
T(r, F) & \leq \frac{7}{2} \bar{N}(r, \infty ; F)+N_{2}(r, 0 ; F)+N_{2}(r, 0 ; G)+\frac{1}{2} \bar{N}(r, 0 ; F)+S(r, f) \\
& \leq \frac{7}{2} \bar{N}(r, \infty ; F)+\frac{3}{2} N_{2}(r, 0 ; F)+N_{2}(r, 0 ; G)+S(r, f) .
\end{aligned}
$$

By the definition of $F, G$ and (6), we have

$$
\begin{aligned}
T(r, f) & \leq \frac{7}{2} \bar{N}(r, \infty ; F)+\frac{3}{2} N_{2}(r, 0 ; F)+N_{2}(r, 0 ; H)+S(r, f) \\
& \leq \frac{7}{2} \bar{N}(r, \infty ; f)+\frac{3}{2} N_{2}(r, 0 ; f)+(\Gamma-d) \bar{N}(r, \infty ; f)+N_{2+\Gamma-d}\left(r, 0 ; f^{d}\right)+S(r, f) .
\end{aligned}
$$

So

$$
\left(\frac{7}{2}+\Gamma-d\right) \Theta(\infty, f)+\frac{3}{2} \delta_{2}(0, f)+d \delta_{2+\Gamma-d}\left(0, f^{d}\right) \leq \Gamma+4,
$$

which contradicts the assumption (3) of Theorem 6.

Subcase 2.3. $k=0$. We know that $F, G$ share " $(1,0)$ ", hence we have

$$
\begin{aligned}
N(r, \infty ; H) & \leq \bar{N}(r, \infty ; F)+\bar{N}(r, 1 ; F \mid \geq 2)+\bar{N}(r, 0 ; F \mid \geq 2)+\bar{N}(r, 0 ; G \mid \geq 2) \\
& +\bar{N}_{L}(r, 1 ; F)+\bar{N}_{L}(r, 1 ; G)+\bar{N}_{0}\left(r, 0 ; F^{\prime}\right)+\bar{N}_{0}\left(r, 0 ; G^{\prime}\right)+S(r, f),
\end{aligned}
$$

and

$$
\begin{gathered}
N_{E}^{1)}(r, 1 ; F)=N_{E}^{1)}(r, 1 ; G)+S(r, f), N_{E}^{(2}(r, 1 ; F)=N_{E}^{(2}(r, 1 ; G)+S(r, f), \\
N_{E}^{1)}(r, 1 ; F) \leq N(r, \infty ; H)+S(r, f) .
\end{gathered}
$$


Using Lemmas 6-8 and (25) and (26), we get

$$
\begin{aligned}
\bar{N}(r, 1 ; F)+\bar{N}(r, 1 ; G) & \leq \bar{N}_{L}(r, 1 ; F)+\bar{N}_{L}(r, 1 ; G)+\bar{N}_{E}^{(2}(r, 1 ; F)+\bar{N}(r, 1 ; G) \\
& \leq N_{E}^{1)}(r, 1 ; F)+N(r, 1 ; G)-\bar{N}_{L}(r, 1 ; G)+\bar{N}_{F>1}(r, 1 ; G)+\bar{N}_{G>1}(r, 1 ; G) \\
& \leq \bar{N}(r, 0 ; F \mid \geq 2)+\bar{N}(r, 0 ; G \mid \geq 2)+\bar{N}(r, \infty ; F)+\bar{N}_{*}(r, 1 ; F, G)+T(r, G) \\
& -m(r, 1 ; G)+O(1)-\bar{N}_{L}(r, 1 ; G)+\bar{N}_{F>1}(r, 1 ; G)+\bar{N}_{G>1}(r, 1 ; G) \\
& +N_{0}\left(r, 0 ; F^{\prime}\right)+N_{0}\left(r, 0 ; G^{\prime}\right)+S(r, F)+S(r, G) .
\end{aligned}
$$

Combining (23) and (27) and by Lemma 2, we can obtain

$$
\begin{aligned}
T(r, f) & \leq 6 \bar{N}(r, \infty ; F)+N_{2}(r, 0 ; F)+2 \bar{N}(r, 0 ; F)+N_{2}(r, 0 ; G)+\bar{N}(r, 0 ; G)+S(r, f) \\
& \leq(6+2 \Gamma-2 d) \bar{N}(r, \infty ; f)++N_{2}(r, 0 ; f)+2 \bar{N}(r, 0 ; f)+N_{2+\Gamma-d}\left(r, 0 ; f^{d}\right) \\
& +N_{1+\Gamma-d}\left(r, 0 ; f^{d}\right)+S(r, f) .
\end{aligned}
$$

So

$$
(6+2 \Gamma-2 d) \Theta(\infty, f)+\delta_{2}(0, f)+2 \Theta(0, f)+d \delta_{1+\Gamma-d}\left(0, f^{d}\right)+d \delta_{2+\Gamma-d}\left(0, f^{d}\right) \leq 2 \Gamma+9,
$$

which contradicts the assumption (4) of Theorem 6 . Now the proof has been completed.

\section{Acknowledgement}

The author (RM) is greatful to the University Grants Commission (UGC), New Delhi, India for Supporting her research work by providing her with a National Fellowship for Higher Education (NFHE).

\section{Competing interests}

The authors declare that they have no competing interests.

\section{Authors' contributions}

All authors have contributed to all parts of the article. All authors read and approved the final manuscript.

\section{References}

[1] Hayman, W. K. Meromorphic functions. Oxford Mathematical Monographs Clarendon Press, Oxford 1964

[2] Lahiri, Indrajit; Sarkar, Arindam. Uniqueness of a meromorphic function and its derivative. JIPAM. J. Inequal. Pure Appl. Math. 5 (2004), no. 1, Article 20, 9 pp.

[3] Liu, Lipei; Gu, Yongxing. Uniqueness of meromorphic functions that share one small function with their derivatives. Kodai Math. J. 27 (2004), no. 3, 272-279.

[4] Li, Nan; Yang, Lianzhong; Liu, Kai. A further result related to a conjecture of R. Brück. Kyungpook Math. J. 56 (2016), no. 2 , 451-464.

[5] Xu, Hong-Yan; Hu, Yi. Uniqueness of meromorphic function and its differential polynomial concerning weakly weighted-sharing. Gen. Math. 19 (2011), no. 3, 101-111.

[6] Yi, Hong-Xun. Uniqueness theorems for meromorphic functions whose $n$th derivatives share the same 1-points. Complex Variables Theory Appl. 34 (1997), no. 4, 421-436.

[7] Yi, Hong-Xun; Yang C.C. Uniqueness theory of meromorphic functions, Science Press, Beijing, (1995).

[8] Yu, Kit-Wing. On entire and meromorphic functions that share small functions with their derivatives. JIPAM. J. Inequal. Pure Appl. Math. 4 (2003), no. 1, Article 21, 7 pp. 